\title{
Satisfação de pais de crianças com necessidades especiais de saúde com os cuidados de enfermagem
}

\author{
Satisfaction of parents of children with special health care needs with nursing care \\ Satisfacción de padres de niños con necesidades especiales de salud con atención de enfermería
}

Recebido: 24/09/2021 | Revisado: 01/10/2021 | Aceito: 10/10/2021 | Publicado: 12/10/2021

\author{
Déborah Viana Oliveira \\ ORCID: https://orcid.org/0000-0001-6321-7598 \\ Faculdades Nova Esperança, Brasil \\ E-mail: deborahviana41@gmail.com \\ Evelyne de Lourdes Neves de Oliveira \\ ORCID: https://orcid.org/0000-0001-7332-2500 \\ Universidade Federal da Paraíba, Brasil \\ E-mail: evelyneoliveira@hotmail.com \\ Jael Rúbia Figueiredo de Sá França \\ ORCID: https://orcid.org/0000-0001-8880-6786 \\ Universidade Federal da Paraíba, Brasil \\ E-mail: jaelrubia@gmail.com \\ Eliane Cristina da Silva Buck \\ ORCID: https://orcid.org/0000-0002-9230-8760 \\ Universidade Federal da Paraíba, Brasil \\ Faculdades Nova Esperança, Brasil \\ E-mail: eliane.buck@academico.ufpb.br
}

\begin{abstract}
Resumo
Introdução: Crianças com necessidades especiais de saúde, geralmente, apresentam limitações físicas, mentais, intelectuais ou sensoriais que exigem de seus cuidadores um acompanhamento diário repleto de atenção, cuidados específicos e habilidades técnicas e humanas. O cuidado realizado de forma holística e integral previne complicações, reduz o estresse e gera na criança e em seus pais sentimentos de satisfação, alegria e gratidão. Objetivo: avaliar a satisfação de pais de crianças com necessidades especiais de saúde com os cuidados de enfermagem. Metodologia: pesquisa quantitativa realizada com 31 pais de crianças internas em um hospital público pediatrico durate o mês de outubro de 2018. Os dados foram coletados mediante formulário e analisados por meio de estatística descritiva. Salienta-se que o estudo foi aprovado pelo Comitê de Ética em Pesquisa sob Protocolo $n^{\circ}$ 151/2018. Resultados: Constatou-se que o nível de satisfação dos pais encontra-se no estrato regular em relação aos cuidados oferecidos pelo enfermeiro às crianças com necessidades especiais de saúde. Entre os domínios analisados, o educacional foi o que apresentou os maiores índices de insatisfação, no qual nenhum quesito desse obteve mediana $>4$ pontos. Verificou-se ainda que os participantes se mostraram insatisfeitos com o hospital, o que pode ter refletido na insatisfação com a assistência do enfermeiro. Conclusão: A satisfação dos pais com os cuidados de enfermagem é um indicador importante da assistencia a criança, podendo ser otimizado por meio da oferta de informações e as orientações de forma clara, com uma linguagem simples e acessível, de maneira respeitosa, gentil e digna.
\end{abstract}

Palavras-chave: Satisfação pessoal; Pais; Criança; Pessoas com necessidades especiais; Cuidados de enfermagem.

\begin{abstract}
Introduction: Children with special health needs usually have physical, mental, intellectual or sensory limitations that demand from their caregivers a daily follow-up full of attention, specific care and technical and human skills. The care provided in a holistic and integral way prevents complications, reduces stress and generates feelings of satisfaction, joy and gratitude in the child and their parents. Objective: to assess the satisfaction of parents of children with special health needs with nursing care. Methodology: quantitative research carried out with 31 parents of children interned in a public pediatric hospital during the month of October 2018. Data were collected using a form and analyzed using descriptive statistics. It should be noted that the study was approved by the Research Ethics Committee under Protocol n. 151/2018. Results: It was found that the level of parental satisfaction is in the regular stratum in relation to the care provided by nurses to children with special health needs. Among the domains analyzed, educational was the one with the highest levels of dissatisfaction, in which none of this item had a median $>4$ points. It was also found that participants were dissatisfied with the hospital, which may have reflected in dissatisfaction with the care provided by nurses. Conclusion: Parental satisfaction with nursing care is an important indicator of child care, which can be optimized by providing clear information and guidance, in simple and accessible language, in a respectful, kind and dignified manner.
\end{abstract}

Keywords: Personal satisfaction; Parents; Child; Disabled persons; Nursing care. 


\begin{abstract}
Resumen
Introducción: Los niños con necesidades especiales de salud suelen tener limitaciones físicas, mentales, intelectuales o sensoriales que demandan de sus cuidadores un seguimiento diario lleno de atención, cuidados específicos y habilidades técnicas y humanas. La atención brindada de manera holística e integral previene complicaciones, reduce el estrés y genera sentimientos de satisfacción, alegría y gratitud en el niño y sus padres. Objetivo: evaluar la satisfacción de los padres de niños con necesidades especiales de salud con los cuidados de enfermería. Metodología: investigación cuantitativa realizada con 31 padres de niños internados en un hospital pediátrico público durante el mes de octubre de 2018. Los datos se recolectaron mediante un formulario y se analizaron mediante estadística descriptiva. Cabe señalar que el estudio fue aprobado por el Comité de Ética en Investigación bajo Protocolo No. 151/2018. Resultados: Se encontró que el nivel de satisfacción de los padres se encuentra en el estrato regular en relación al cuidado brindado por enfermeras a los niños con necesidades especiales de salud. Entre los dominios analizados, el educativo fue el que presentó mayores niveles de insatisfacción, en el que ninguno de este ítem tuvo una mediana> 4 puntos. También se encontró que los participantes estaban insatisfechos con el hospital, lo que puede haberse reflejado en una insatisfacción con la atención brindada por las enfermeras. Conclusión: La satisfacción de los padres con los cuidados de enfermería es un indicador importante del cuidado infantil, que puede optimizarse proporcionando información y orientación claras, en un lenguaje sencillo y accesible, de manera respetuosa, amable y digna.
\end{abstract}

Palabras clave: Satisfacción personal; Padres; Niño; Personas con discapacidad; Atención de enfermería.

\title{
1. Introdução
}

No âmbito da saúde, a população infanto-juvenil devido suas características peculiares e o processo de crescimento e desenvolvimento sempre apresentou maiores necessidades de cuidados se comparados aos adultos. Contudo, uma parcela dessa população requer cuidados ainda mais amplos, seja decorrente do aumento da demanda, frequência ou da complexidade destes, seja pelas necessidades de tecnologias assistivas que possibilitem a manutenção da vida ou a melhora da qualidade de vida dessas crianças vistas como "especiais" (Arrué, et al., 2016, Huang, Freed, \& Dalziel, 2020).

O termo "criança especial" surgiu nos Estados Unidos com a definição pela Maternal and Child Health Bureau, do conceito Children with special health care needs (CSHCN) traduzido no Brasil como Crianças com Necessidades Especiais de Saúde (CRIANES). Esta expressão é utilizada para designar crianças que apresentam algum risco ou que já vivenciam condições que provoquem limitações físicas, de desenvolvimento, comportamental ou emocional que necessitem de cuidados e serviços de saúde em demandas maiores do que a média dessa população perdurando por mais de 12 meses (Arrué, et al., 2016, Huang, Freed, \& Dalziel, 2020).

Nos países em desenvolvimento, as CRIANES representam entre 13 e 18\% da população infanto-juvenil, contudo no Brasil, não se tem dados quantitativo sobre essa. No entanto, segundo dados da última Pesquisa Nacional de Saúde cerca de $8,4 \%$ da população brasileira apresentavam algum tipo de deficiência, e dentre estes 5,2\% eram crianças e adolescentes entre 2 e 17 anos de idade (Huang, Freed, \& Dalziel, 2020, IBGE, 2021). Compreende-se, portanto, que as CRIANES, na realidade fazem parte de uma parcela da população que precisa de cuidados singulares, tendo em vista as limitações que as mesmas estão sujeitas, principalmente no que competem as ações de saúde.

Além das dificuldades relacionadas a condição clínica da criança como dificuldade de definição diagnóstica, tratamento e internações recorrente, as CRIANES e suas famílias ainda enfrentam diariamente diversos desafios no que tange a garantia de direitos, vulnerabilidade social, escassez de políticas públicas específicas, deficiência de infraestrutura na rede de atenção à saúde, despreparo dos profissionais em lidar com essas crianças e suas famílias em todos os níveis de atenção a saúde, a precariedade nas redes de apoio, além do preconceito e estigmatização social (Góes \& Cabral, 2017).

Com o objetivo de minimizar esses desafios, o Ministério da Saúde instituiu, em 2002, a Política Nacional de Saúde da Pessoa com Deficiência e, em 2011, o Plano Nacional dos Direitos da Pessoa com Deficiência - Plano Viver sem Limite. Estes visam integrar políticas e estratégias entre diversos setores da sociedade para garantir os direitos das pessoas com deficiências, incluindo as CRIANES e, principalmente no âmbito da saúde (Brasil, 2002, Brasil, 2011). Assim, articulou-se entre todos os níveis de atenção à saúde uma Rede de Cuidados a Pessoa com Deficiência como forma de operacionalizar essas 
políticas, tornar os serviços mais acessíveis, ampliar e qualificar a assistência de saúde como foco em prevenção de doenças, identificação precoce das deficiências, e reabilitação intelectual, visual, auditiva, física, ostomia e múltiplas deficiências (Brasil, 2012).

Além disso, as CRIANES necessitam de cuidados integrais e contínuos ofertados por uma equipe interdisciplinar que detenham além de conhecimentos e habilidades no manejo da condição clínica, possuam também tempo, atenção e sensibilidade para perceber as necessidades da criança e de seus pais, bem como suas limitações e dificuldades (Inácio \& Peixoto, 2017). É nesse cenário que a equipe de enfermagem assume um papel de destaque, principalmente durante o período de hospitalização, acolhendo a criança e seus familiares, estimulando o envolvimento deste no processo saúde-doença-cuidado da criança e auxiliando os pais a se adaptarem as novas necessidades do filho.

Nessa perspectiva, a equipe de enfermagem precisa ter atitudes que demonstrem confiança, empatia, respeito e advocacia, sendo um modelo de parceria em relações estabelecidas entre os pais e os demais profissionais de saúde (Alves, Amendoeira, \& Charepe, 2017). Saber reconhecer a importância da presença da família nos cuidados à criança e valorizar a família no contexto do cuidado é indispensável.

Diante disso, torna-se importante reafirmar que a presença e a participação dos pais são essenciais para o tratamento da criança, desde o momento das primeiras manifestações clínicas e da definição do diagnóstico médico até a estabilidade da doença e autonomia da criança, quando possível. No âmbito hospitalar essa participação precisa ser efetiva, desde a admissão até a alta da criança, pois todas as informações precisam ser repassadas corretamente de ambos os lados: profissionais e responsáveis pela mesma (Barbosa, Balieiro, \& Mandetta, 2012). Nesse sentido, a atuação do enfermeiro deve ser pautada na integralidade do cuidar das CRIANES. Essas necessidades, quando atendidas, geram satisfação tanto na criança quanto nos pais. Sendo assim, a satisfação dos pais com a assistência de enfermagem ofertada é um indicador de qualidade desse cuidado.

Dessa forma a visão da família sobre os serviços de saúde, em especial do cuidado de enfermagem, é transformada de acordo com as decisões tomadas em face da melhoria dos cuidados ofertados, sendo assim usado como um ato motivador para incentivar um cuidado maior com as crianças, buscando não apenas oferecer cuidado, mas melhorar sua qualidade de vida (Azevêdo, Júnior, \& Crepaldi, 2017). Assim, a satisfação dos pais de CRIANES é um indicador de qualidade da assistência oferecida, sendo, por isso, importante ser investigado. Logo, este estudo trouxe como objetivo: avaliar a satisfação de pais de crianças com necessidades especiais de saúde com os cuidados de enfermagem.

\section{Metodologia}

Estudo descritivo, de abordagem quantitativa realizada em um hospital referência no atendimento de crianças, localizado no município de João Pessoa-Paraíba. A população do estudo foi constituída por todos os pais de crianças com necessidades especiais internadas nos setores de clínica médica, clínica cirúrgica e as três unidades de terapia intensiva: geral, neurológica e cardiológica.

Neste contexto, considerou-se os seguintes valores como base para o cálculo amostral: população $=407$ referente ao número de acompanhantes de crianças com deficiência neurológica ou neuropatia internadas no mês de abril de 2018; erro amostral $=5 \%$; nível de confiança $=95 \%$; proporção de $0,8 \%(\mathrm{p}=0,008)$ que corresponde ao percentual da população brasileira acometida por algum tipo de deficiência intelectual ou neurológica (IBGE, 2015). Logo, o resultado obtido foi $=12$ que, ao considerar um acréscimo de $20 \%$ de perdas (0.2), totalizou uma amostra de 14 participantes. Contudo, durante a coleta de dados conseguiu-se extrapolar o número de voluntários definidos no cálculo amostral, sendo a amostra final constituída por 31 participantes.

Para seleção da amostra, foram adotados como critérios de elegibilidade: ser pai ou mãe de CRIANE com faixa etária entre 0 e 17 anos de idade, estar acompanhando a criança no momento da coleta de dados, que a criança tenha diagnóstico 
médico confirmado de deficiência neurológica ou neuropatia, que a criança estivesse interna no serviço há pelo menos 48h; e como critérios de exclusão: mães ou pais com idade menor que 18 anos, ou que possuíssem alguma dificuldade cognitiva e intelectual que as limitassem ou as impedissem de compreender os questionamentos para a coleta dos dados. Critérios de descontinuidade: participantes que demonstrassem o desejo de desistir de participar do estudo, uma vez que já tenha sido iniciado.

Para a realização da coleta de dados, foi utilizado um instrumento em forma de formulário organizado em três partes. A primeira detalha as características dos pais e comtempla as variáveis: sexo, idade, nível educacional, profissão e situação profissional. A segunda, refere-se à aplicação do Instrumento de Satisfação do Paciente -ISP. A terceira corresponde a uma escala de satisfação do paciente com o hospital.

O Patient Satisfaction Instrument (ISP) é uma escala tipo Likert que busca avaliar a satisfação dos pacientes com os cuidados de enfermagem tendo sido adaptado e validado para a cultura brasileira. A escala comtempla 25 quesitos, com questões positivas e negativas, estruturadas em três domínios, a saber: Técnico-profissional, sendo avaliada a competência do enfermeiro; A confiança, a qual vai avaliar as características do enfermeiro; Aspectos da comunicação e educacional, visando à habilidade do enfermeiro em fornecer informações e orientações (Moglia Junior \& Lopes, 2018).

O ISP traz como score de satisfação do paciente a média das pontuações obtidas em cada quesito, onde valores $\leq 2$ considera-se insatisfeito, $=3$ satisfação regular e $\geq 4$ satisfeito com o atendimento recebido. No Brasil, o ISP já foi utilizado em várias pesquisas com diferentes tipos de populações, a destacar o estudo de realizado com mães de crianças em um alojamento conjunto. E apesar desse instrumento não ter sido inicialmente desenvolvido pensando nesta população sua aplicação no referido estudo demonstrou uma satisfação de 3,7 na escala (1-5) (Moglia Junior \& Lopes, 2018).

Os dados foram coletados durante o mês de setembro de 2018, após a aprovação do estudo pelo Comitê de Ética em Pesquisa sob protocolo $\mathrm{n}^{\circ}$ 151/2018. A análise dos dados foi realizada por meio de estatística utilizando para tanto o programa estatístico Statistical Package for Social Sciences (SPSS) - versão 21 e o programa Bioestat versão 5.4. O somatório das variáveis ordinais dos instrumentos ISP foram comparados usando ANOVA 1-Fator entre as 3 (três) dimensões. Dentro de cada dimensão os dados foram comparados usando o teste não-paramétrico de Mann-Whitney entre grau de parentesco (pai ou mãe); faixa etária (até 30 anos ou até 40 anos); nível de escolaridade (ensino fundamental ou médio); situação empregatícia (empregado ou desempregado) e, para nível de conhecimento sobre o cuidar (nenhum, pouco ou muito), foi usado o teste nãoparamétrico de Krukal-Wallis.

Para as variáveis ordinais do instrumento da Avaliação Geral do Hospital (AGH), além dos testes não paramétricos de Mann-Whitney e de Krukal-Wallis para comparação entre grau de parentesco; faixa etária; nível de escolaridade; situação empregatícia e nível de conhecimento sobre o cuidar, também foi calculado o alfa de Cronbach, para verificar a consistência interna do questionário. Nesse estudo, não foi possível calcular o alfa de Cronbach para a escala ISP, pois o tamanho da amostra versus o número de questões tornou o uso dessa medida inviável. Os resultados são mostrados na forma de gráficos e tabelas. Para todas as análises o nível de significância foi de 5\%.

\section{Resultados}

Os participantes da amostra foram constituídos por 22 (71\%) mães e 9 (29\%) pais. Com relação a faixa etária dos pais, $19(61,3 \%)$ tinham idades entre 18 e 30 anos, enquanto que $12(38,7 \%)$ tinham até 40 anos. A maioria dos participantes, 17 (54,8\%), possuíam o ensino médio completo enquanto que 14 (45,2\%) concluíram apenas o ensino fundamental. Com relação ao conhecimento da patologia que levou a criança a hospitalização, 20 (64,5\%) participantes afirmaram terem pouco conhecimento, $5(16,1 \%)$ referiram que não tinham nenhum conhecimento e $6(19,4 \%)$ afirmaram possuir um conhecimento amplo sobre a doença do filho. Quando investigados sobre a situação empregatícia, 11 (35,5\%) participantes referiram estar 
com vínculo empregatício enquanto que 20 (64,5\%) afirmaram estar desempregados e dentre esses 8 (25,8\%) indicaram que sua principal ocupação era dona de casa.

Com relação ao filho com necessidades especiais que encontrava-se interno no momento da coleta de dados, verificou-se que as idades variaram entre 1 ano e 17 dias a 16 anos, sendo $7(22,6 \%)$ crianças abaixo de 1 ano de idade, 18 (58\%) entre 1 e 5 anos, $3(9,7 \%)$ entre 6 e 10 anos e $3(9,7 \%)$ com idade acima de 10 anos. Contudo, todos os participantes referiram ter outro filho, além do que estava hospitalizado, e por isso observou-se uma mediana de 2 filhos por participantes, onde a maior parte, 14 (45\%), destes comunicaram que cuidavam de crianças menores de um ano, enquanto 11 (35,7\%) referiram que cuidavam de crianças entre 2 e 5 anos de idades, $6(19,4 \%)$ referiram cuidar de crianças acima de 6 anos de idade. Com relação ao cuidado com a CRIANE, 5 (16,1\%) participantes relataram que cuidavam sozinhos dos filhos, enquanto $26(83,9 \%)$ referiram que partilhavam os cuidados com outras pessoas, a saber: irmã, avó, tia e tio.

A Tabela 1 abaixo apresenta o percentual de resposta das questões com pontos positivos segundo os domínios do instrumento, onde $\mathrm{n}$ representa o número de participantes, \% o percentual de cada resposta, DP o Desvio Padrão e M a Mediana, indicando a satisfação dos participantes com os cuidados de enfermagem ofertados a seus filhos durante a hospitalização.

Tabela 1 - Distribuição descritiva e de tendência central das respostas dos participantes por atributos positivos, segundo domínio ( $\mathrm{N}=31)$. João Pessoa-PB, setembro de 2018.

\begin{tabular}{|c|c|c|c|c|c|c|c|c|c|c|c|c|}
\hline \multirow{3}{*}{ Questão } & \multicolumn{10}{|c|}{ Respostas } & \multirow{3}{*}{$\mathbf{M}$} & \multirow{3}{*}{ DP } \\
\hline & \multicolumn{2}{|l|}{1} & \multicolumn{2}{|l|}{2} & \multicolumn{2}{|l|}{3} & \multicolumn{2}{|l|}{4} & \multicolumn{2}{|l|}{5} & & \\
\hline & $\mathbf{N}$ & $\%$ & $\mathbf{N}$ & $\%$ & $\mathbf{n}$ & $\%$ & $\mathbf{N}$ & $\%$ & $\mathbf{N}$ & $\%$ & & \\
\hline \multicolumn{13}{|c|}{ Domínio tecnico profissional } \\
\hline 01 & 0 & 0 & 0 & 0 & 0 & 0 & 18 & 58 & 13 & 42 & 4,4 & $\pm 0,5$ \\
\hline 03 & 7 & 22,5 & 5 & 16 & 2 & 6,4 & 2 & 6,4 & 15 & 48,3 & 3,4 & $\pm 1,7$ \\
\hline 04 & 0 & 0 & 7 & 22,5 & 3 & 9,6 & 9 & 29 & 12 & 38,7 & 3,8 & $\pm 3,8$ \\
\hline 07 & 0 & 0 & 0 & 0 & 7 & 22,5 & 13 & 42 & 11 & 35,4 & 4,1 & $\pm 4,1$ \\
\hline \multicolumn{13}{|c|}{ Domínio confiança } \\
\hline 16 & 6 & 19,3 & 0 & 0 & 3 & 9,6 & 7 & 22,5 & 15 & 48,3 & 3,8 & $\pm 1,5$ \\
\hline 17 & 2 & 6,4 & 4 & 12,9 & 2 & 6,4 & 12 & 38,7 & 10 & 32,2 & 3,8 & $\pm 1,2$ \\
\hline 19 & 1 & 3,2 & 12 & 38,7 & 5 & 16 & 5 & 16,1 & 6 & 19,3 & 3,1 & $\pm 1,2$ \\
\hline 20 & 0 & 0 & 0 & 0 & 0 & 0 & 9 & 29 & 22 & 71 & 4,7 & $\pm 0,5$ \\
\hline 22 & 3 & 9,6 & 12 & 38,7 & 0 & 0 & 6 & 19,3 & 10 & 32,2 & 3,3 & $\pm 1,5$ \\
\hline 25 & 1 & 3,2 & 7 & 22,5 & 11 & 35,4 & 12 & 38,7 & 0 & 0 & 3,1 & $\pm 0,9$ \\
\hline \multicolumn{13}{|c|}{ Domínio educacional } \\
\hline 9 & 2 & 6,4 & 3 & 9,6 & 7 & 22,5 & 12 & 38,7 & 7 & 22,5 & 3,6 & $\pm 1,1$ \\
\hline 12 & 2 & 6,4 & 11 & 35,4 & 3 & 9,6 & 5 & 16 & 9 & 29 & 3,3 & $\pm 1,4$ \\
\hline 13 & 0 & 0 & 0 & 0 & 5 & 16 & 11 & 35,4 & 14 & 45,1 & 4,2 & $\pm 0,8$ \\
\hline 14 & 16 & 51,6 & 15 & 48,3 & 0 & 0 & 0 & 0 & 0 & 0 & 1,5 & $\pm 0,5$ \\
\hline
\end{tabular}

*1 Discordo totalmente; 2 Discordo; 3 Não tenho Opinião; 4 Concordo; 5 Concordo Totalmente. Fonte: Dados da pesquisa (2018). 
Entre os dados apresentados na tabela 1, foi observado que no domínio técnico-profissional a primeira questão obteve o maior percentual (100\%) de satisfação quando somado as respostas "concordo" e "concordo totalmente", seguidas pela questão sete (7) ( 77,5\%). Foi possível verificar ainda que apenas em ambas as questões o valor da mediana foi superior a quatro (4), indicando, portanto, um alto nível de satisfação dos pais com os cuidados de enfermagem no que se refere a preocupação dos pais em seguir corretamente as orientações dos médicos acerca dos cuidados e tratamentos com a criança, bem como a habilidade do enfermeiro em auxiliar o médico durante possíveis procedimentos.

Contudo, ao analisar apenas as repostas "concordo totalmente", pôde-se evidenciar que o terceiro quesito, o qual indaga "Os profissionais da equipe de enfermagem dão bons conselhos?", obteve o maior percentual, apesar da sua mediana estar abaixo de quatro pontos. Isto indica que os participantes acreditam que os profissionais de enfermagem os escutam e os compreendem ao ponto de dar opiniões e conselhos que lhes são válidos. Assim, entendem-se que a atenção, a empatia e a formação de vínculos com as crianças e com seus pais foram essenciais para que os profissionais de enfermagem pudessem criar um importante canal de diálogo, ao ponto de seus conselhos serem considerados benéficos pelos participantes.

No domínio confiança, apenas a vigésima questão que indaga "quando eu preciso conversar com alguém eu posso contar meus problemas ao enfermeiro (a) obteve uma mediana acima de quatro pontos. Isto indica que os pais sentem confiança para dialogar, até mesmo aspectos íntimos de sua vida social e familiar com o enfermeiro. Para isso, é necessário que se tenha formado um vínculo entre o profissional e os pais, uma relação mútua de respeito e confiança com o objetivo mútuo de cuidar e promover o bem-estar a criança. Entretanto, os quesitos que obtiveram os maiores percentuais de positividade, quando somadas as repostas "concordo" e "concordo totalmente", foram respectivamente o de número 20 (100\%), 17 (70,9\%) e $16(70,8 \%)$.

As questões de número 17 e 16 referiam-se, respectivamente, sobre "sentir-se a vontade para fazer perguntas ao enfermeiro" e "o enfermeiro é uma pessoa agradável de se ter por perto". Tais afirmativas evidenciam que os pais enxergam o enfermeiro como uma pessoa boa, o que o faz se sentir seguro para esclarecer dúvidas acerca da doença e tratamento da criança, bem como aspectos da vida social desta. Assim, a atenção dispensada pelo enfermeiro para ouvir os pais e a criança, e conseguir ser resolutivo em suas ações, o faz ser visto como uma pessoa bem quista e agradável na opinião dos participantes. Tal resultado corrobora como o encontrado em um estudo realizado no Hospital Universitário da Universidade Federal do Maranhão no município de São Luís, o qual também evidenciou um alto nível de segurança e confiança no enfermeiro por parte dos pacientes (Santos, Sardinha, \& Santos, 2017).

Torna-se importante destacar que em relação à insatisfação demonstrada nas repostas apresentadas na tabela 1, as questões 19 e 25 foram as que obtiverem menores valores de mediana. Contudo, a questão 22 foi a que demonstrou os maiores níveis de insatisfação dos participantes como os cuidados do enfermeiro dispensados a seus filhos, representando cerca de metade das respostas, quando somados os itens "discordo" e "discordo totalmente". Estas afirmativas referem-se a capacidade do enfermeiro de compreender os sentimentos da criança e de seus acompanhantes, e saber lidar com estes com compaixão.

Assim, quando o enfermeiro tem essa capacidade diminuída, isso pode acarretar prejuízos no desenvolvimento da empatia, no fortalecimento do vínculo e na dificuldade de se estabelecer uma comunicação efetiva. Tais resultados, representam uma ambiguidade com os dados apresentados, mostrando uma relação de dualidade e fragilidade nas relações de comunicação.

Em relação ao domínio educacional, apenas o quesito de número 13 apresentou pontuação de mediana acima de quatro. Neste, o participante afirma que o enfermeiro fornece orientações na velocidade correta. Sendo assim, passíveis de terem suas palavras compreendidas. Entretanto, referem na questão número 14, de modo expressivo (100\%), que o enfermeiro não fornece explicações completas e suficientes do porquê os exames foram solicitados a à criança. Logo, nota-se um prejuízo na comunicação entre o profissional e os pais da criança, sendo necessário que as informações sobre os procedimentos sejam 
informadas de maneira completa, clara e honesta. Na Tabela 2 nenhum dos quesitos, nos três domínios, atingiram uma pontuação média > 4. Isto indica um grau de insatisfação maior do que o de satisfação em todos os quesitos.

Tabela 2 - Distribuição descritiva e de tendência central das respostas dos participantes por atributos negativos, segundo domínio (N = 31). João Pessoa-PB, setembro de 2018.

\begin{tabular}{|c|c|c|c|c|c|c|c|c|c|c|c|c|}
\hline \multirow{3}{*}{ Questão } & \multicolumn{10}{|c|}{ Respostas } & \multirow{3}{*}{$\mathbf{M}$} & \multirow{3}{*}{ DP } \\
\hline & \multicolumn{2}{|l|}{1} & \multicolumn{2}{|l|}{2} & \multicolumn{2}{|l|}{3} & \multicolumn{2}{|l|}{4} & \multicolumn{2}{|l|}{5} & & \\
\hline & $\mathbf{N}$ & $\%$ & $\mathbf{N}$ & $\%$ & $\mathbf{n}$ & $\%$ & $\mathbf{N}$ & $\%$ & $\mathbf{N}$ & $\%$ & & \\
\hline \multicolumn{13}{|c|}{ Domínio tecnico profissional } \\
\hline $\mathbf{0 2}$ & 3 & 9,6 & 14 & 45,1 & 1 & 3,2 & 9 & 29 & 4 & 13 & 2,9 & $\pm 1,3$ \\
\hline 05 & 2 & 6,4 & 10 & 32,2 & 14 & 45,1 & 4 & 13 & 1 & 3,2 & 2,7 & $\pm 0,9$ \\
\hline 06 & 0 & 0 & 2 & 6,4 & 13 & 42 & 15 & 48,3 & 0 & 0 & 3,4 & $\pm 0,6$ \\
\hline \multicolumn{13}{|c|}{ Domínio confiança } \\
\hline 15 & 20 & 64,5 & 1 & 3,2 & 1 & 3,2 & 7 & 22,5 & 2 & 6,4 & 2,0 & $\pm 1,5$ \\
\hline 18 & 15 & 48,3 & 6 & 19,3 & 1 & 3,2 & 4 & 13 & 5 & 16,1 & 2,3 & $\pm 1,6$ \\
\hline 21 & 7 & 22,5 & 8 & 26 & 6 & 19,3 & 7 & 22,5 & 3 & 9,6 & 2,7 & $\pm 1,3$ \\
\hline 23 & 1 & 3,2 & 10 & 32,2 & 13 & 42 & 6 & 19,3 & 1 & 3,2 & 2,9 & $\pm 0,9$ \\
\hline 24 & 4 & 13 & 11 & 35,4 & 7 & 22,5 & 6 & 19,3 & 2 & 6,4 & 2,7 & $\pm 1,1$ \\
\hline \multicolumn{13}{|c|}{ Dominio educacional } \\
\hline 8 & 14 & 45,1 & 1 & 3,2 & 7 & 22,5 & 4 & 13 & 5 & 16,1 & 2,5 & $\pm 1,6$ \\
\hline 10 & 2 & 6,4 & 12 & 39 & 2 & 6,4 & 7 & 22,5 & 7 & 22,5 & 3,2 & $\pm 1,4$ \\
\hline 11 & 19 & 61,2 & 1 & 3,2 & 0 & 0 & 3 & 9,6 & 7 & 22,5 & 2,3 & $\pm 1,8$ \\
\hline
\end{tabular}

*1 Concorda Totalmente; 2 Concordo; 3 Não tenho opinião; 4 Discordo; 5 Discordo Totalmente. Fonte: Dados da pesquisa (2018).

No domínio técnico-profissional, evidencia-se um alto nível de insatisfação no que se refere a organização e a calma do enfermeiro no desenvolver de suas atividades diárias, como evidencia a afirmativa de número 2, a qual obteve um percentual de 54,7\% de insatisfação, quando somadas as respostas "concordo" e "concordo totalmente". A questão de número 5 também apresentou uma expressividade considerável de insatisfação, na qual aproximadamente 39\% dos participantes consideraram o enfermeiro muito lento para fazer as ações de cuidado, contrapondo, apenas $16 \%$ a consideraram satisfatória neste aspecto. Contudo, neste quesito, $45 \%$ dos participantes referiram não possuir uma opinião formada sobre o assunto.

Dentre os quesitos do domínio técnico-profissional, o único que apresentou um bom nível de satisfação, cerca de 50\% das respostas, foi a questão 6, que referia que os enfermeiros não fazem corretamente o seu trabalho. Contudo, se observar atentamente a tabela 3, o que chama a atenção são os outros $42 \%$ dos participantes que referiram não saber opinar. Seria tal resposta um indicativo de medo dos participantes por algum tipo de represália ou prejuízo da assistência a seus filhos, que eles pensariam sofrer em caso de uma avaliação "ruim" do enfermeiro ou um desconhecimento acerca dos cuidados que devem ser dispensados as suas crianças, e por isso a insuficiência de parâmetros para equiparar a assistência ideal com a realizada pela equipe. Um estudo realizado em um hospital no Rio de Janeiro com 300 pacientes, ao utilizar o ISP, também obteve como resultado na questão 6 um bom nível de satisfação com o cuidado de enfermagem (Santos, et al., 2018).

No domínio confiança, o quesito de número 15 que relata que "o enfermeiro deveria ser mais atencioso do que é" seguido pelo de número 18 que refere "o enfermeiro deveria ser mais amigável do que é", foram os que expressaram os 
maiores níveis de insatisfação dos pais com os cuidados de enfermagem. Evidenciando, assim, a necessidade de mais atenção e comunicação da equipe no que diz respeito a importar-se com a criança e demostrar isso de uma forma mais cordial, respeitosa, levando em consideração a vivência e fragilidades, da família e na criança, no decorrer da hospitalização.

Dentre todos os questionamentos do domínio confiança, o que obteve o maior percentual de satisfação - 32,1\%, foi quesito 21 que referia que o enfermeiro estaria muito ocupado para perder seu tempo com a criança e seus pais. Isso indica que ainda há muito o que melhorar neste aspecto da assistência do enfermeiro às crianças com necessidades especiais de saúde, nas perspectivas de seus pais.

No domínio educacional, a questão 11 seguida pela de número 8 foi a que expressou os maiores índices de insatisfação, apresentando percentuais de 64,4\% e 48,3\%, respectivamente. Na pergunta de número 11: "Eu gostaria que os profissionais da equipe de enfermagem me dessem mais informações sobre os resultados do meu exame", houve um alto índice de responsáveis insatisfeitos com a equipe, pois necessitam das informações sobre a saúde dos seus filhos, uma vez que as incertezas são geradoras de ansiedade e sentimentos negativos. A questão de número 8 dizia que: "O enfermeiro(a) muitas vezes acha que você não é capaz de entender explicação médica sobre a sua doença, então ele(a) simplesmente não se preocupa em explicar", havendo um percentual chamativo de insatisfação dos pais com relação as informações que não são explicadas de maneira simples sendo ela a comunicação entre os pais e o enfermeiro, na perspectiva de conversar numa linguagem simples e clara, bem como trazer bem-estar ao participante e a criança durante o diálogo, tendo este, então, como um importante instrumento para alcançar a satisfação dos pais.

Em geral, pode-se constatar, a partir dos resultados obtidos, que o nível de satisfação dos pais com os cuidados oferecidos pelo enfermeiro às crianças com necessidades especiais de saúde, encontrava-se regular, uma vez que a média geral das respostas encontradas neste estudo foi de $+3,2$, estando, entretanto, abaixo do score esperado, 4. Portanto, é visível a necessidade da assistência a essas crianças precisa ser melhorada, principalmente no que se refere a ações de caráter educativo.

Para avaliar a satisfação dos participantes em relação aos serviços hospitalares foi utilizada a Escala de Avaliação Geral do Hospital. Para esta foi calculado o alfa de Cronbach, para verificar a consistência interna do questionário, o qual evidenciou o valor $\alpha=0,98$. Neste contexto, pode-se afirmar que o resultado encontrado neste estudo indica um alto grau de consistência interna dos dados do AGH e, portanto, um excelente grau de confiabilidade.

Comparando todas as respostas dos itens avaliados no instrumento de $\mathrm{AGH}$, através do teste não-paramétrico de Kruskal-Wallis $(\mathrm{H}=0,48 ; \mathrm{p}=0,99 ; \mathrm{gl}=6)$, não foi observada diferença estatística entre as respostas. Tal fato, indica que os participantes consideraram o atendimento da instituição hospitalar pesquisada como estando em nível médio de satisfação (de 3 ou um pouco acima) que pode ser observado na Tabela 3 a seguir, refletindo um descontentamento com o atendimento hospitalar.

Tabela 3 - Estatísticas descritiva dos itens da Avaliação Geral do Hospital (AGH) com os 31 responsáveis dos pacientes investigados no Complexo Pediátrico Arlinda Marques, João Pessoa, setembro de 2018. (n=31).

\begin{tabular}{cccccccc}
\hline Medidas descritivas & AGH1 & AGH2 & AGH3 & AGH4 & AGH5 & AGH6 & AGH7 \\
\hline Mínimo & 1 & 1 & 1 & 1 & 1 & 1 & 1 \\
Máximo & 5 & 5 & 5 & 5 & 5 & 5 & 5 \\
Amplitude Total & 4 & 4 & 4 & 4 & 4 & 4 & 4 \\
Média Aritmética & 3.2 & 3.2 & 3.1 & 3.2 & 3.2 & 3.1 & 2.9 \\
Desvio Padrão & 2.0 & 2.0 & 2.0 & 2.0 & 2.0 & 2.0 & 2.0 \\
Erro Padrão & 0.4 & 0.4 & 0.4 & 0.4 & 0.4 & 0.4 & 0.4 \\
Coeficiente de Variação & $63.4 \%$ & $63.4 \%$ & $66.3 \%$ & $63.4 \%$ & $63.4 \%$ & $66.3 \%$ & $69.2 \%$ \\
\hline
\end{tabular}


Fonte: Dados da pesquisa (2018).

Quando estratificados por subgrupos para as variáveis demografias: grau de parentesco (pai ou mãe); faixa etária (até 30 anos ou até 40 anos); grau de escolaridade (ensino fundamental ou médio); situação empregatícia (empregado ou desempregado); nível de conhecimento sobre o cuidar (nenhum, pouco ou muito), os resultados das respostas dos entrevistados não mostraram diferença estatística significativa em nenhum dos 7 itens avaliados (Tabela 4).

Tabela 4 - Valores de significância (p-valor) dos testes de comparação entre os itens do instrumento AGH para os subgrupos das variáveis demográficas com os 31 responsáveis dos pacientes investigados no Complexo Pediátrico Arlinda Marques, João Pessoa, 2018. $(\mathrm{n}=31)$.

\begin{tabular}{cccccccc}
\hline $\begin{array}{c}\text { Teste de Mann-Whitney ou } \\
\text { Kruskal-Wallis }\end{array}$ & AGH1 & AGH2 & AGH3 & AGH4 & AGH5 & AGH6 & AGH7 \\
\hline Grau de parentesco & 0,54 & 0,54 & 0,69 & 0,54 & 0,54 & 0,69 & 0,81 \\
Faixa etária & 0,73 & 0,73 & 0,92 & 0,73 & 0,79 & 0,92 & 0,92 \\
Grau de escolaridade & 0,69 & 0,69 & 0,89 & 0,69 & 0,32 & 0,47 & 0,65 \\
Situação empregatícia & 0,66 & 0,67 & 0,68 & 0,69 & 0,46 & 0,47 & 0,48 \\
Nível de conhecimento & 0,51 & 0,52 & 0,17 & 0,51 & 0,95 & 0,61 & 0,23 \\
sobre cuidar & & & & & & & \\
\hline
\end{tabular}

Fonte: Dados da pesquisa (2018).

\section{Discussão}

Tradicionalmente, no seio familiar as mulheres exercem a função de senhoras do lar e cuidadoras dos filhos. Seja como chefe de família ou não, a mulher toma para si a responsabilidade de cuidar, unir e zelar pelo bem-estar da sua família, ainda mais quando, além de mulher, ela também é mãe. Na busca pela qualidade de vida do filho, a mãe assume o pilar central da estrutura familiar, e é sob a sua administração que estão a criação, a educação e a saúde dos filhos (Miranda, Oliveira, Toia, \& Stucchi, 2015).

A atribuição de cuidadora é um ponto de vista que a família tem da mãe, e que ela tem de si mesma. Para desempenhar tal competência a mãe acaba criando meios, como o ajustamento do horário de trabalho ou a renúncia do emprego em favor das rotinas domésticas e dos cuidados direcionados ao filho (Oliveira, et al., 2017). Assim, a maternagem requer da mãe uma doação diária e constante, que se intensifica quando a criança é acometida por alguma doença ou condição crônica que limite a sua vida e o seu bem-estar. Quando acometidas por distúrbios neurológios e neuropatias, a criança necessita ainda mais de atenção e zelo, além de cuidados específicos para sua condição.

Quando a criança nasce com algum distúrbio neurológico que limite o seu crescimento e/ou o desenvolvimento de forma adequada, a família é surpreendida por uma série de situações das quais não tem domínio. As suas expectativas dão lugar a preocupação com a vida da criança, as incertezas e os medos surgem. Quando de forma crônica, a condição da criança pode ocasionar uma desorganização e uma reestruturação familiar, onde a mãe, geralmente, abarca para si a totalidade dos cuidados ao filho.

Assim como este, vários estudos evidenciaram que quando os filhos são acometidos por alguma condição crônica, a mãe abarca para si a função de cuidadora principal, tendo dificuldade de partilhar o cuidado, seja em domicilio, seja no hospital. Esta situação, geralmente, relaciona-se ao sentimento de luto pelo filho idealizado, culpa pelo adoecimento da criança, a carência de apoio e preconceito familiar e social, assim como autopreconceito e autocobrança em demasia, entre 
outros fatores. O abandono do seu emprego, também é listado como um fator contribuinte para a dificuldade de compartilhar os cuidados da criança, uma vez que devido às dificuldades financeira, o pai da criança tende a aumentar sua da carga de trabalho (Santos \& Miranda, 2016, Oliveira, et al., 2017, Freitag, Milbrath, \& Motta, 2020).

A família tem um papel importante no desenvolvimento da criança. Por isto, o cuidado à a uma criança com necessidade especial é bastante complexo e frágil, tendo em vista que muitos pais não esperam ter filhos com alguma limitação, mostrando muitas vezes não ter habilidade alguma no cuidado à criança. Durante a coleta de dados, foram inúmeros os relatos dos pais referindo que tinham pouco conhecimento sobre a patologia dos seus filhos e que não sabiam como lidar em alguma emergência. Assim, a inclusão da família no processo de cuidado do paciente depende não só da forma como a instituição concebe ações de acompanhamento para a família, mas também, da maneira como a gestão da equipe de enfermagem integra a família dentro da organização dos procedimentos, e de como equipe e família se relacionam no acompanhamento e cuidado ao paciente internado, cuidado este que deve abranger a dimensão psicossocial (Santos \& Miranda, 2016, Santos, et al., 2018a).

Dentre as patologias apresentadas pelas crianças que estavam internas, às que se destacaram pela alta prevalência foram os distúrbios convulsivos/epilepsia. No Brasil, a incidência de epilepsia na infância é de 1 a $2 \%$ entre crianças com distúrbios neurológicos. Junto com a convulsão febril, elas se caracterizam como a causa mais comum de convulsão em crianças entre 6 meses e 5 anos (Vilela, Brízida, Pádua, Silva, \& Ferreira, 2019). Tais patologias geram medo e estigma, até mesmo entre os parentes mais próximos, associando-se a carência de conhecimento sobre a doença pelos pais das crianças, em que muitos deles afirmam não saber lidar com o ocorrido e procuravam rapidamente um hospital.

Estudo acerca da percepção dos pais sobre a epilepsia realizado no setor de neurologia de um hospital em Sorocaba concluiu que os participantes/pais tinham um conhecimento adequado sobre o assunto, contudo persistiam aspectos negativos na compreensão do distúrbio (Fonseca, Sandro, \& Silva, 2018). Ações de educação em saúde, nesses casos, se fazem importantes para esclarecer dúvidas onde outrora os pais não sabem ou tenham vergonha de perguntar, bem como evitar os estigmas sobre a doença da criança.

Os resultados também demonstraram que a maior parte dos participantes se encontrava na faixa etária entre 30 e 40 anos, e a grande maioria tinha um nível de escolaridade regular, uma vez que tinham completado o Ensino Médio. Contudo, houve alguns participantes que concluíram apenas o Ensino Fundamental. Vale ressaltar que nenhum dos responsáveis possuía nível de escolaridade técnico ou superior.

Esse estudo mostrou que muitos pais das crianças hospitalizadas estão desempregados, esse alto número de desemprego pode ser associado a tal situação pelo aumento das necessidades de cuidados que demandam mais tempo e atenção à criança. A relação do cuidado, junto com a relação maternal entre seu filho especial, faz com que aumente para si a responsabilidade da assistência, em que exige, muitas vezes, o abandono do emprego. As Intervenções diárias com a criança, junto com a responsabilidade dentro da própria casa, ocupam todo tempo dos pais, provocando uma alta porcentagem de desemprego e dificuldades financeiras (Constantinidis, Silva, \& Ribeiro, 2018).

Diante dessa amostra é possível observar que a maioria dos pais divide os cuidados com as crianças, em que a mesma é compartilhada com algum parente como avós, tias e irmãs sendo isso necessário para o abastecimento financeiro para manutenção dos tratamentos das crianças. Ademais, o compartilhamento de cuidados minimiza a sobrecarga materna sendo por isso importante quando outros membros da família fornecem apoio.

O Instrumento de Satisfação do Paciente- ISP foi realizado com os pais/responsáveis de crianças com necessidades especiais que se encontravam hospitalizadas, tendo como foco saber até onde está a satisfação dos mesmos com os cuidados da equipe de enfermagem. Tendo em vista que as crianças não possuem sua própria autonomia. 
A criança vivencia a hospitalização como uma experiência que não passa despercebida, e muito menos para seus familiares ou acompanhantes. Uma vez que o processo de hospitalização ocorre com elas a reflexão deve ser redobrada, à medida que esta ação pode comprometer seu desenvolvimento mental. Levando a sensação de estranhamento ao ambiente hospitalar e a sensação de abandono, quando a função de cuidar não é desempenhada, e hospitalizações prolongadas, o hospital é visto como local de proibições, podendo contribuir para comportamentos desadaptados da criança (Santos \& Miranda, 2016). A perspectiva de levar os pais para dentro do hospital traz alterações nas relações de trabalho estabelecidas no ambiente hospitalar. Estes são encorajados a ficar com os filhos durante a hospitalização, tornando-se mais um dos agentes no processo de cuidado.

Durante o processo de avaliação dos dados foram evidenciados que o tempo de internação faz com que se crie um vínculo de confiança, respeito e empatia entre a família, na qual a mesma forma um elo forte entre profissional e usuário, garantindo segurança no que concerne ao atendimento de suas necessidades (Santos, et al., 2018b). A equipe de enfermagem realizando o cuidado tem um contato direto, havendo assim um olhar humanístico naquela internação. Para os pais, o cuidado, atenção e a recepção de uma boa equipe ao seu filho é um aspecto positivo, promovendo uma compreensão ampliada do processo saúde-doença e dos pontos de vulnerabilidade. A importância de se tornar um ambiente acolhedor dentre as dificuldades vistas, pode ter como consequência tensão, conflito e relação frágil entre familiares e equipe de saúde, sendo possível minimizar os pontos negativos que virão no decorrer dos dias hospitalizado.

Entretanto, muitos dos pais relatam que não existe uma comunicação mútua entre os familiares e a equipe, visto que pode se trazer preocupações dos procedimentos a serem feitos, levando assim ao medo, apreensão e ansiedade da família podendo ocorrer a incerteza sobre o prognóstico, a falta de privacidade e de individualidade, ao ambiente desconhecido e, por vezes, aterrorizante, pois sentem a necessidade de uma escuta qualificada, onde requer uma maior atenção (Inácio \& Peixoto, 2017).

Existindo um déficit na comunicação, pode levar essa equipe a um modelo biomédico de cuidados, observando apenas a parte técnica e não levando em conta o psicológico e social daquela família, podendo haver uma mudança na perspectiva do cuidado entre família e equipe de enfermagem. No entanto, a enfermagem tem consciência da sua responsabilidade diante da qualidade da assistência prestada ao paciente, pois existem leis, normas e éticas da profissão as quais requerem o cuidado e a atenção nos procedimentos e cuidado, como também ajuda na área da social e psíquica do atendimento à saúde (Freitas, Silva, Minamisava, Bezerra, \& Sousa, 2014).

A evolução dos modelos de assistência de enfermagem à criança hospitalizada teve uma mudança significativa, tendo como princípio uma abordagem modelo centrada na criança e um modelo centrado na criança e sua família. Sendo na primeira abordagem com foco apenas na criança tem como um todo o esforço da equipe de saúde, um foco na obtenção de dados referentes aos problemas de saúde da mesma, no qual o diagnóstico rápido tem medidas alcançadas e um conceito nas ações terapêuticas. No modelo com foco na criança e sua família, a internação é vista não apenas como um agravo psicológico à criança, mas também como possível trauma à família, em que necessitam do apoio da equipe de saúde (Miranda, Oliveira, Toia, \& Stucchi, 2015).

Nos dias atuais, o cuidado à saúde não se limita apenas ao corpo biológico, mas estende-se ao apoio emocional, social e de desenvolvimento, com o objetivo de atender à criança e à família em sua totalidade, gerando um olhar holístico e humanizado, sem apenas observar a doença. Apresentando esse novo modelo, reservar o respeito e a dignidade entre os profissionais e os familiares, a partilha de informações e a participação da família nas tomadas de decisões sobre o processo de cuidado.

Sendo assim, o fornecimento de informações completas, corretas e claras sobre as condições para o tratamento de uma criança, em que verificar como os pais compreendem a situação e o tratamento da mesma é de responsabilidade da equipe de 
enfermagem. A troca de informações com a família é um meio de compartilhar sem julgar as reações, realizando os cuidados de forma conjunta, agrupando as observações dos familiares no plano de cuidado (Santos \& Miranda, 2016).

Além de informações relacionadas ao quadro clínico da criança, procedimentos diagnósticos e tratamentos, se faz importante que a enfermagem também disponha aos familiares informações sobre os direitos fundamentais que são garantidos a essa população pelo Estatuto da Criança e do Adolescente e pelo Plano Viver sem Limite, os benefícios sociais que podem ser requeridos, bem como instruir sobre a Rede de Cuidados a Pessoa com Deficiência com enfoque nos Centros Especializado em Reabilitação, uma vez que este possibilitam a integralidade da assistência em todos os níveis da atenção à saúde e melhorar a qualidade de vida dessas criança.

A insatisfação dos pais/responsáveis com a estrutura do hospital é um influenciador na satisfação do paciente com relação ao tratamento, sejam eles recursos humanos, físicos, estruturais ou tecnológicos. Expondo, assim, a insatisfação do cuidado nos recursos, criando uma dificuldade no trabalho do enfermeiro, podendo fazê-lo se sentir sobrecarregado e não conseguir prestar uma boa assistência, levando os pacientes e familiares que utilizam do serviço a não se sentiram satisfeitos.

\section{Considerações Finais}

Foi observado neste estudo que, os pais de crianças com necessidades especiais de saúde apresentaram um nível regular de satisfação com os cuidados realizados pelos enfermeiros(as) a seus filhos em todos os domínios do ISP. Destaca-se, no entanto, que as ações de caráter educativo foram as que apresentaram os menores valores de satisfação, evidenciando, assim, a necessidade de melhorar as informações e as orientações que são dispensadas às crianças e aos seus pais, fazendo-as de forma clara, com uma linguagem simples e acessível, de maneira respeitosa, gentil e digna. Essa comunicação, ainda deve ser realizada em um momento e um local adequados, respeitando o tempo de compreensão da criança e de seus pais, bem como a garantia de que as dúvidas existentes sejam esclarecidas e o acesso ao enfermeiro seja livre de qualquer receio, medo ou preconceito.

É importante ressaltar que os pais/responsáveis também necessitam de uma atenção, tendo assim o direito de serem assistidos por uma equipe multiprofissional durante o período de hospitalização do filho. Além disso, mesmo que a mãe seja, a responsável principal pelo cuidar, ter outros membros da família que a ajudem no desenvolvimento do cuidado se faz primordial para garantir o seu descanso físico, mental e emocional, apoio social-econômico e até mesmo espiritual. Portanto, faz-se importante que o enfermeiro, ao cuidar de uma criança com necessidades especiais de saúde, estimule a interação e o apoio familiar aos pais, de modo a possibilitar o fortalecimento de vínculos, enfrentamento da hospitalização de uma forma mais tranquila e a reinserção da criança e de seu cuidador de forma mais gradual no seu ciclo social, familiar e até mesmo educacional.

Deste modo, o estudo identificou a satisfação dos pais de crianças com necessidades especiais de saúde, sendo observados os cuidados que eram prestados enquanto seus filhos encontravam-se hospitalizados, alcançando, desta maneira, os objetivos propostos. Vale ressaltar que houve limitações no decorrer da pesquisa, tais como as explicações de respostas de determinadas questões do instrumento, como o porquê os pais não opinaram em algumas questões, mostrando ter um alto índice de percentual. Assim, seria interessante que pesquisas que utilizem o ISF também utilizem um roteiro de entrevista semiestruturada para complementar.

\section{Referências}

Alves, J., Amendoeira, J., \& CHarepe, Z. (2017). A parceria de cuidados pelo olhar dos pais de crianças com necessidades especiais de saúde. Revista gaúcha enfermagem, 38 (4), 1-7. http://dx.doi.org/10.1590/1983-1447.2017.04.2016-0070.

Arrué, A. M., Neves, E. T., Magnago, T. S., Cabral, I. E., Gama, S. G., \& Hökerberg., Y. H. (2016). Tradução e adaptação do children with special. Caderno 
de Saúde Pública, 32 (6), e00130215, 1-7. https://doi.org/10.1590/0102-311X00130215

Azevêdo, A. V., Júnior, A. C., \& Crepaldi, M. A. (2017). Interação equipe de enfermagem, família, e criança hospitalizada: revisão integrativa. Ciência saúde coletiva, 22 (11), 3653-3666. https://doi.org/10.1590/1413-812320172211.26362015

Barbosa, M. A., Balieiro, M. M., \& Mandetta, M. A. (2012). Cuidado centrado na família no contexto da criança com deficiência e sua família: uma análise reflexiva. Texto Contexto Enfermagem, 21 (1), 194-199. https://doi.org/10.1590/S0104-07072012000100022

Brasil. (2002). Portaria n ${ }^{\circ}$ 1060, de 5 de junho de 2002. Ministério da saúde. https://bvsms.saude.gov.br/bvs/saudelegis/gm/2002/prt1060_05_06_2002.html

Brasil. (2011). Decreto $n^{\circ}$ 7.612, de 17 de novembro de 2011. Retrieved from http://www.planalto.gov.br/ccivil_03/_ato2011-2014/2011/decreto/d7612.htm

Brasil. (2012). Portaria no 793, de 24 de abril de 2012. Ministério da saúde. https://bvsms.saude.gov.br/bvs/saudelegis/gm/2012/prt0793_24_04_2012.html

Constantinidis, T. C., Silva, L. C., \& Ribeiro, M. C. (2018). "Todo mundo quer ter um filho perfeito":vivências de mães de crianças com autismo. Psico-USF, 23 (1), 47-58. https://doi.org/10.1590/1413-82712018230105

Fonseca, M. S., S. B., \& Silva, B. G. (2018). Percepção dos pais sobre a epilepsia. Revista da Faculdade de Ciências Médicas de Sorocaba, 20 (1), 33-7. https://doi.org/10.23925/1984-4840.2018v20i1a8

Freitag, V. L., Milbrath, V. M., \& Motta, M. G. C. (2020) Tornar-se mãe de uma criança com paralisia cerebral: sentimentos vivenciados. Psicoloia em estudos, 25, e41608, 1-14. https://doi.org/10.4025/psicolestud.v25i0.41608

Freitas, J. S., Silva, A. E., Minamisava, R., Bezerra, A. L., \& Sousa, M. R. (2014). Qualidade dos cuidados de enfermagem e satisfação do paciente atendido em um hospital de ensino. Revista Latino-Americana de Enfermagem, 22 (3), 454-460. https://doi.org/10.1590/0104-1169.3241.2437

Góes, F. G., \& Cabral, I. E. (2017). Hospital discharge in children with special health care needs and its different dimensions. Revista de Enfermagem da UERJ, 25, e18684, 1-6. http://dx.doi.org/10.12957/reuerj.2017.18684

Huang, L., Freed, G. L., \& Dalziel, K. (2020). Children with special health care needs: how special are their health care needs? Academic Pediatrics, 20 (8), 1109-1115. https://doi.org/10.1016/j.acap.2020.01.007

Inácio, A., \& Peixoto, A. (2017). A assistência de enfermagem e o cuidado familiar às crianças com necessidades especiais de saúde: uma revisão integrativa. Revista Atençao em Saúde, 15 (53), 87-94. https://doi.org/10.13037/ras.vol15n53.4593

Instituto Brasileiro de Geografia e Estatítica. (2015). IBGE. Pesquisa nacional de saúde: 2013. ciclos de vida. Brasil e grandes regiões. IBGE.

Instituto Brasileiro de Geografia e Estatítica. (2021). Pesquisa nacional de saúde : 2019 : ciclos de vida : Brasil. IBGE.

Miranda, A. R., Oliveira, A. R., Toia, L. M., \& Stucchi, H. K. (2015). A evolução dos modelos de assistência de enfermagem à criança hospitalizada nos últimos trinta anos: do modelo centrado na doença ao modelo centrado na criança e família. Revista da Faculdade de Ciências Médicas de Sorocaba, 17 (1), 5 9. https://revistas.pucsp.br/index.php/RFCMS/article/view/12890

Moglia Junior, J. B., \& Lopes, E. L. (2018). Escalas concorrentes para mensuração da satisfação do paciente hospitalar. Revista de Gestão e Secretariado, 9 (3),103-130. https://doi.org/10.7769/gesec.v9i3.776

Oliveira, E. L. N., Silva, E. C., Silva, M. F. O. C., Coutinho, S. E. D., Sales, A. M. C., Souza, A. T. O., Andrade, S. P., \& França, J. R. F. S. (2017). Mothers' perception on recreational activities in the treatment of childhood cancer. International Archives of Medicine, 10 (121). https://doi.org/10.3823/2391

Santos, B. A., Milbrath, V. M., Freitag, V. L., Nunes, N. J. S., Gabatz, R. I. B., \& Silva, M. S. (2018a). The impact of cerebral palsy diagnosis from the perspective of the family. Revista Mineira de Enfermagem. 23,e-1187, 1-7. https://doi.org/10.5935/1415-2762.20190035

Santos, F. D., Gomes, A. M., Rafael, R. D., Silva, F. V., Marques, S. C., \& Cunha, L. P. (2018b). The patients' satisfaction with nursing care in hemodialysis. Revista de pesquisa cuidado é fundamental online, 10 (2), 432-440. https://doi.org/10.9789/2175-5361.2018.v10i2.432-440

Santos, M. A., Sardinha, A. H., \& Santos, L. N. (2017). Satisfação dos usuários com os cuidados dos enfermeiros. Revista Gaúcha de Enfermagem, 38 (1), e57506, 1-7. https://doi.org/10.1590/1983-1447.2017.01.57506

Santos, R. C., \& Miranda, F. A. (2016). Importância do vínculo entre profissional-usuário na estratégia de saúde da família. Revista Enfermagem UFSM , 6 (3): 350-359. https://doi.org/10.5902/2179769217313

Silveira, A., \& Neves, E. (2011). Crianças com necessidades especiais de saúde: tendências das pesquisas em enfermagem. Revista de Enfermaem UFSM, 1 (2), 1-7. https://doi.org/10.5902/217976922500

Vilela, L. R., Brízida, L. V., Pádua, M. d., Silva, B. M., \& Ferreira, L. S. (2019). Perfil de crianças e adolescentes internados por distúrbiosneurológicos. Residência Pediátrica, 9 (3), 1-5. https://doi.org/10.25060/residpediatr 